\title{
Response to: Determination of Any Correlation between Sagittal Spinopelvic Configuration and Progressive Collapse of Acute Osteoporotic Compression Spine Fractures: A Retrospective Radiological Analysis
}

\author{
Yossi Smorgick $^{1,2}$, Alex Geftler ${ }^{3}$, Sergey Goldstein ${ }^{2,4}$, Yigal Mirovsky ${ }^{2,4}$, Ronen Blecher ${ }^{2,4}$, Yoram Anekstein ${ }^{1,2}$ \\ ${ }^{1}$ Spine Unit, Assaf Harofeh Medical Center, Zerifin, Israel \\ ${ }^{2}$ Department of Orthopedic Surgery, Sackler Faculty of Medicine, Tel-Aviv University, Tel-Aviv, Israel \\ ${ }^{3}$ Department of Orthopedic Surgery, Soroka Medical Center, Ben Gurion University, Beer Sheva, Israel \\ ${ }^{4}$ Department of Orthopedic Surgery, Assaf Harofeh Medical Center, Zerifin, Israel
}

We read with great interest the comments that were sent to us regarding our study, "Determination of any correlation between sagittal spinopelvic configuration and progressive collapse of acute osteoporotic compression spine fractures: a retrospective radiological analysis [1]."

The authors' comment that we did not analyze the sagittal parameters and that we did not mention the neurological status of the patient at the time of presentation in the selection criteria. These are two excellent comments and we should have used the term spinopelvic and should have stated that all patients included in the study were all neurologically intact.

In another comment, the authors write that there was no mention of the level of injury in the inclusion criteria and that we did not mention how patients with cervical and upper thoracic fractures were managed at our center. We would like to refer the authors to Table 2 in page 4 which specify the level of injury in all cases and to page 2 the second paragraph were we state that the study in- cluded only thoracolumbar fracture: "we retrospectively identified all patients treated for thoracolumbar fractures in our institution between January 2008 and July 2013, through a computerized search of International Classification."

The authors would like to know how the pathological fractures were diagnosed using advanced imaging techniques such as magnetic resonance imaging (MRI) scans which are more sensitive in diagnosing them. We do not routinely do an MRI scan for each compression fracture. In the cases in which the clinical scenario or radiological findings implied for pathological fracture, an MRI scan was done but these patients were excluded from the study.

The authors were interested in how we confirmed that the fractures are acute without doing an MRI or a bone scan. We do not routinely do a bone scan or an MRI for each compression fracture. An experienced radiologist or spine surgeon can in the right clinical setting distinguish between acute and old fracture, especially with a 3-month

Received Aug 29, 2020; Accepted Sep 3, 2020

Corresponding author: Yossi Smorgick

The Spine Unit, Assaf Harofeh Medical Center, Zerifin 70300, Israel

Tel: +972-8-9779437, Fax: +972-8-9779434, E-mail: ysmorgick@gmail.com 
follow-up.

The authors wrote that we did not mention the position in which radiographs were taken. We thank them for this comment. The initial radiographs were done in the supine position, while during follow-up patients underwent standing weight-bearing radiographs.

To conclude, we thank the authors for their important comments.

\section{Conflict of Interest}

No potential conflict of interest relevant to this article was reported.

\section{References}

1. Smorgick Y, Geftler A, Goldstein S, Mirovsky Y, Blecher R, Anekstein Y. Determination of any correlation between sagittal spinopelvic configuration and progressive collapse of acute osteoporotic compression spine fractures: a retrospective radiological analysis. Asian Spine J 2020 Jan 8 [Epub]. https://doi. org/10.31616/asj.2019.0139. 